\title{
Dental Care and the COVID-19 Pandemic: The Precautionary Principle and the Best Available Evidence
}

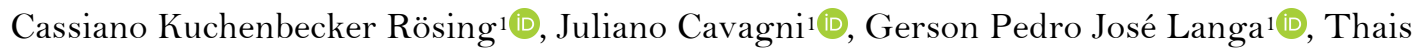

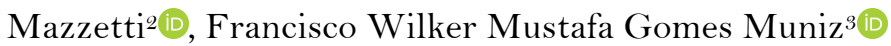

\begin{abstract}
${ }^{1}$ Department of Periodontology, Faculty of Dentistry, Federal University of Rio Grande do Sul, Porto Alegre, RS, Brazil. ${ }^{2}$ Post-graduation Program in Dentistry, Federal University of Pelotas, Pelotas, RS, Brazil.

${ }^{3}$ Department of Periodontology, Faculty of Dentistry, Federal University of Pelotas, Pelotas, RS, Brazil.
\end{abstract}

Author to whom correspondence should be addressed: Cassiano Kuchenbecker Rösing, Faculty of Dentistry, Department of Periodontology, Federal University of Rio Grande do Sul, Rua Ramiro Barcelos, 2492, Porto Alegre, RS, Brazil. 90040-060. Phone: +55 5133085318. E-mail: ckrosing@hotmail.com.

Academic Editor: Yuri Wanderley Cavalcanti

Received: 02 June 2020 / Accepted: 15 June 2020 / Published: 22 June 2020

How to cite this article: Rösing CK, Cavagni J, Langa GPJ, Mazzetti T, Muniz FWMG. Dental care and the COVID-19 pandemic: the precautionary principle and the best available evidence. Pesqui Bras Odontopediatria Clín Integr. 2020; 2O(supp1):e0115. https://doi.org/10.1590/pboci.2020.118

\begin{abstract}
The precautionary principle is part of evidence-based healthcare and is used both preventively and therapeutically when there is no available evidence about how to manage problems/diseases/conditions that are especially life-threatening. However, since it is not always based in the most qualified evidence, it is frequently questioned. The emergence of a highly contagious disease, with increased levels of morbimortality, an acute respiratory syndrome, the so called Coronavirus Disease 2019 (COVID-19), led health professionals to look for the best alternatives to save lives. In this sense, the precautionary principle was evocated. The aim of this paper is to make a reflection about the precautionary principle, the dental profession and COVID-19. It is important to have in mind that in such a disease, guidelines, protocols and approaches can change very fast, since a continuous evaluation of all policies is mandatory. During the pandemic, elective procedures may be restricted, but international organizations removed the recommendation to postpone elective procedures. Clinicians are advised to be updated about their local current policies. On the other hand, there are cases in which in-office dental care is unavoidable, such as individuals with pain, spontaneous bleeding and dental trauma. Biosafety is upmost importance when seeking patients during pandemic. In this sense, it was concluded that precautionary principle should be, therefore, used. However, caution needs to be taken and continuous surveillance necessary.
\end{abstract}

Keywords: Coronavirus; Dental Health Services; Evidence-Based Practice. 


\section{Introduction}

Evidence-based healthcare has been considered the current paradigm for all health professions [1]. This movement started with evidence-based medicine and spread for all health approaches. In such paradigm, the best available evidence should be used to treat individuals. This means that the so-called authority-based healthcare is increasingly questioned. Dentistry has a considerable difference from Medicine, since the majority of the situations faced by the profession does not have death as an outcome. Death is undoubtedly a true outcome. In Dentistry, with some exceptions, tooth loss is one of the most typical true outcomes [2]. Of course, patient-related outcome measures are also true outcomes (e.g., masticatory function, esthetics, oral health-related quality of life, among others) and have to be always considered.

The paradigm of evidence-based dentistry combines three fundamental aspects: the best available evidence, the expertise of the professional and patient values and preferences. All of these aspects should be taken into consideration in the decision-making processes.

However, there are some situations in which the best available evidence is of low quality or, even, inexistent. In 2020, a pandemic emerged: the Coronavirus Disease 2019 (COVID-19). It has been responsible for a very high number of deaths worldwide. The contamination with the SARS-CoV-2 (coronavirus) generates a strong respiratory syndrome, which, especially for older adults and for those with chronic diseases, has a high mortality rate [3]. Therefore, different preventive and therapeutic measures have been advocated. Some of the measures are not based on high-quality evidence and others might even not have any support. Their indication is frequently based in the so-called precautionary principle [4]. The present article aims at making a deep thought about the precautionary principle in dental care, especially taking into consideration the COVID-19 pandemic.

\section{The Precautionary Principle - Definition and Reflections}

The precautionary principle is a preventive concept. Traditionally, it comes from epidemiological studies that identify potential risk factors and, even without the complete proof, start to be part of the preventive policies [4]. It is used when the evidence neither clearly supports nor refutes the hypothesis. Of course, the inclusion of action on the preventive policy needs to be under continuous and close surveillance, up to the moment in which the evidence supports or discards the measure. Figure 1 summarizes the precautionary principle in the context of evidence-based healthcare.

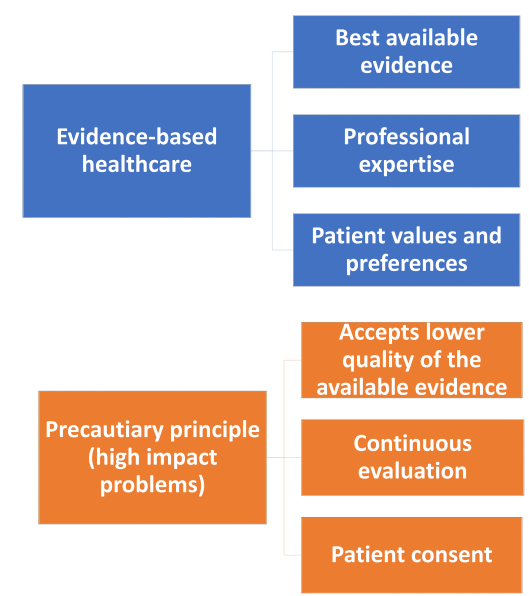

Figure 1. Summary of the evidence-based healthcare and precautionary principle. 
A very important situation in the use of the precautionary principle is that adverse events are also taken into consideration [5]. Moreover, the nature and morbimortality of the situation under consideration is of utmost importance. The precautionary principle is much more useful in situations in which morbimortality is high, which is specifically the case with COVID-19 [4].

In addition to preventive measurements, the precautionary principle is useful in therapeutic approaches. For example, in some types of cancer, therapy starts to be used even before statistically significant differences are observed in comparison to a control group [6]. However, in such situations, in the same manner, that in preventive approaches, a close surveillance of the positive and negative effects is mandatory.

It is very important that the one's understanding, advocating or even analyzing the precautionary principle are aware of the fact that uncertainty is present in these situations in various levels [4]. Evidencebased healthcare tries to rule out uncertainty with the best possible means; however, when life is threatened, higher levels of uncertainty need to be tolerated.

In this respect, one situation that needs to be strengthened is that the "absence of evidence is not evidence of absence". This means that when you do not have evidence in a specific situation, it does not support that this situation is useless or does not work [5]. This is a very popular epidemiological sentence that needs to be always taken into consideration both in the decision-making process as well as in the planning and censuring of research projects. Sometimes it is not ethical not to perform a study, if there is no evidence to it, just because of an individual's belief. So, the precautionary principle is also used with proposals that will be accepted or refuted if continued under adequate surveillance [5].

On the other hand, one very important situation comes with the precautionary principle. This is: which is the outcome of the problem/disease/condition? If the outcome is death, high levels of handicap, or similar situations, evidence of less quality might support the approaches until a definitive answer is achieved [4]. However, this is not adequate if the problem/disease/condition does not have a very harmful outcome. In Dentistry, the precautionary principle is, therefore, very limited [5]. One argument frequently comes into the picture: "if it does not harm, why not to use it?". This is completely inadequate in evidence-based healthcare unless specific reasons are raised to use the precautionary principle [4].

Several points of view are extremely critical to the precautionary principle, especially due to the misuse of it. Some reports even put it in a perspective of a scientific fallacy or incoherence [7]. Clearly, this is not a situation that has only one side. A more comprehensive understanding of the principle is mandatory, with a critical analysis of every situation involving the precautionary principle [6].

\section{An Analysis of COVID-19}

The pandemic of COVID-19 probably started in China and has spread worldwide [8]. This is a highly contagious disease that inputs high levels of uncertainty for healthcare professionals. This is mainly related to the fact that it is a new disease and lack of knowledge exacerbates the situation.

The present study was written between May 20th and June 5th, 2020. This is of high importance to be highlighted, since even during this 2-week interval, several situations changed and the information is being updated sometimes more than once a day. It is known that the pandemic has reached all continents of the world and that in absolute numbers of death, the United States of America and Brazil are the countries with high figures [9,10]. It is estimated that more than 370 thousand people died worldwide up to the end of May 2020 and that the number of infected people is of more than 6.2 million [11]. One study estimated that the prevalence of risk factors to severe COVID-19 range between $34 \%$ to $54.4 \%$ among Brazilian adults, when 
different criteria are considered [12]. Moreover, São Paulo (range: 35.6\% to 58.2\%), Rio de Janeiro (range: $36 \%$ to $55.8 \%$ ), Minas Gerais (range: $35.6 \%$ to $55.1 \%$ ) and Rio Grande do Sul (range: $39.5 \%$ to $58.4 \%$ ) are the Brazilian states that demonstrated the highest prevalence of at least one risk factor for severe COVID-19 [12].

Research is emerging from different countries and it seems that keeping social distance is one of the measurements that decreases infectivity [13]. This is also of importance, since a flattening of the curve may be possible, allowing the health systems to get prepared to it. Together with the uncertainty of any kind of approach comes the need for governmental investment worldwide, which elicits high levels of polemics.

The World Health Organization is trying to lead the understanding process of the pandemic and has already declared that we are facing a public health emergency, with the need of conjunct efforts from every citizen of every country [11]. Based on the precautionary principle (since no evidence was available for this specific disease), different protective measures utilized collateral evidence trying to protect the populations and the continuous evaluation of such measurements constitutes the current best available evidence.

As in any situation in evidence-based healthcare, the methods used to evaluate anything are essential. In this respect, data on prevalence is not available. This is due to the fact that different countries have different protocols for testing individuals, different ways of counting for death causes, etc. Probably, a significant number of deaths by COVID-19 were not reported as such, especially due to under notification or even due to lack of testing of the dead individuals.

Some countries with more established healthcare systems have better forms of notifications. For example, in the United States of America, a public-private partnership for community-based testing is happening [14]. In Germany, there is a private partnership with laboratories and all healthcare facilities to provide as much COVID-19 tests as possible [15].

One point that needs to be analyzed is that associations between some measures and elevation or flattening of the disease curve are available. Social isolation has been demonstrated to be effective [13]. The use of masks has been proposed [16], but the World Health Organization does not recommend the use of mask for healthy individuals, except for health workers or for individuals with direct contact with a sick person [17]. CDC recommends the use of cloth face coverings associated with hand washing, or hand sanitizer with at least $60 \%$ alcohol, social distancing (at least $1.8 \mathrm{~m}$ ), clean and disinfection of surfaces [18].

Another interesting situation that cannot be forgotten is that a hypothesis of a possible positive effect of chloroquine and hydroxychloroquine in patients with COVID-19 was raised. This was the first hypothesis that led to a wrong understanding by parts of the healthcare systems. If in one hand a possible interesting effect was demonstrated, on the other the evidence was very weak and mainly observational. After some weeks of usage by those that understood the potential benefits would be worth, studies are not confirming such benefit. On the contrary, it seems that the posed risks are very high [19]. Therefore, in a very short period of time, higher quality evidence transformed a possibility of treatment in an inadequate approach.

The reality is that we are facing an unknown disease, with a high level of mortality and that direct qualified evidence is not available neither for prevention nor for treatment. In this respect, taking into consideration the idea of the precautionary principle, measures based on collateral evidence might be taken and need continuous evaluation in order to, if proven consistently beneficial, become part of established protocols and, in case of not a proven benefit or a demonstration of important adverse effects, to be banned from the strategies. 


\section{The Precautionary Principle, COVID-19 and Oral Care}

In the context of COVID-19, Dentistry is facing a new challenge. Similarly to professionals that work in intubation of infected patients, dental professionals deal with an important situation regarding aerosol. Saliva is contaminated and a source of infection both for health professionals as well as for other patients that are in the same environment. The literature also suggests that salivary glands could be a potential reservoir for the virus [20].

Also, it should be highlighted that elective procedures should be limited at this point in the pandemic. This means that maintenance visits, for example, are probably postponed. Despite that, Center of Diseases Control and Prevention (CDC) (USA) removed the recommendation to postpone elective procedures in May 18th [21]. In Brazil, there are different recommendations, varying for each state. Clinicians must be updated about the local current policies [22,23]. They all depend on the numbers of infected people and the capacity of the health system.

One important information that has to be given for the population is that oral hygiene methods should be even emphasized, in order to avoid, to the best possible means, the necessity of urgent dental care or, even, deterioration of oral health [24].

On the other hand, there are cases in which in-office dental care is unavoidable, such as individuals with pain, spontaneous bleeding, dental trauma, etc. [24]. In these situations, biosafety is fundamental. In this respect, Dentistry has a lot to teach other professions. However, traditional biosafety measurements are probably not enough. Hence, evidence-based approaches should be preferred. Moreover, part of the decisions will not be evidence-based or, at least, not based on the best quality evidence. But one has to decide and, taking into consideration the best available direct or collateral evidence, guidelines should be constructed.

Different organizations have worked on this topic and, up to the moment of the present article, some recommendations were made by them. $\mathrm{CDC}$ and $\mathrm{ADA}$ recommendations include previous contact with the patients for COVID-19 screening, avoid non-emergent dental care when the patient has COVID-19 symptoms, limit the number of companions whenever possible, minimize the number of patients in the waiting room and check the patient's temperature before dental care [21,25].

One rapid systematic review identified produced guidance and reports that presented recommendations from international organizations and professional bodies on the current requirements for the re-opening of dental services [26]. This study summarized that face mask and eye wear protection (glasses/googles, face shields) are indicated to all staff as personal protective equipment (PPE) [26]. The use of dedicated working uniform and single use gloves are also pointed out. Regarding post-operative cleaning, it is recommended that all surfaces must be cleaned and disinfected following every patient, and the clinical floor must be cleaned from 2 to 3 times per day [26]. This disinfection may be performed with hypochlorite/chlorine based solutions or with 60-70\% alcohol. Furthermore, clinical waste must be disposed of as per regulations of local system. The disposal of PPE and other disposable contaminated materials are recommended to be placed in a hard-lid container [26].

One example of the included suggested procedures is rinsing with $\mathrm{H}_{2} \mathrm{O} 2$. For this suggestion, direct evidence is not available. The potential of $\mathrm{H}_{2} \mathrm{O} 2$ in reducing viral load is an example of the use of the precautionary principle. However, this needs continuous evaluation.

Since evidence-based Dentistry also relies on patient participation, it is very important to clearly inform the individuals seeking dental care about the whole situation and have it in a signed written informed consent. 


\section{Conclusion}

In the moment of the writing of the present article, very little high-quality evidence was available for prevention and treatment of COVID-19. The precautionary principle should be, therefore, used. However, caution needs to be taken and continuous surveillance necessary. Science has to be mature to take steps further and steps back, with the main aim of healthcare, which is the well-being of humanity.

\section{Authors' Contributions}

\begin{tabular}{|c|c|c|}
\hline CKR & (D) $0000-0002-8499-5759$ & $\begin{array}{l}\text { Conceptualization and Writing - Original Draft Preparation and Writing - } \\
\text { Review and Editing. }\end{array}$ \\
\hline $\mathrm{JC}$ & (D) $0000-0003-0062-6604$ & Conceptualization and Writing - Original Draft Preparation. \\
\hline GPJL & (iD) $0000-0002-5900-6330$ & Conceptualization and Writing - Original Draft Preparation. \\
\hline $\mathrm{TM}$ & (D) $0000-0001-6877-2099$ & Conceptualization and Writing - Original Draft Preparation. \\
\hline FWGM & (i) $0000-0002-3945-1752$ & Conceptualization and Writing - Original Draft Preparation. \\
\hline
\end{tabular}

\section{Financial Support}

This study was financed in part by the Coordenação de Aperfeiçoamento de Pessoal de Nível Superior - Brasil (CAPES) Finance Code 001

\section{Conflict of Interest}

The authors declare no conflicts of interest.

\section{References}

[1] Dodson TB. Evidence-based medicine: its role in the modern practice and teaching of dentistry. Oral Surg Oral Med Oral Pathol Oral Radiol Endod 1997; 83(2):192-7. https://doi.org/10.1016/s1079-2 104(97)90005-6.

[2] Hujoel PP, DeRouen TA. A survey of endpoint characteristics in periodontal clinical trials published 1988-1992, and implications for future studies. J Clin Periodontol 1995; 22(5):397-407. https://doi.org/10.1111/j.1600-051x.1995.tbo0167.x

[3] Wang X, Fang X, Cai Z, Wu X, Gao X, Min J, et al. Comorbid chronic diseases and acute organ injuries are strongly correlated with disease severity and mortality among COVID-19 patients: a systemic review and meta-analysis. Research 2020; 2020:2402961. https://doi.org/10.34133/2020/2402961

[4] Resnik DB. The precautionary principle and medical decision making. J Med Philos 2004; 29(3):281-99. https://doi.org/10.1080/03605310490500509

[5] Tickner J, Coffin M. What does the precautionary principle mean for evidence-based dentistry? J Evid Based Dent Pract 2006; 6(1):6-15. https://doi.org/10.1016/j.jebdp.2005.12.006

[6] Weiss NS. When can the result of epidemiologic research not eliminate the need to invoke the precautionary principle? J Evid Based Dent Pract 2006; 6(1):16-8. https://doi.org/10.1016/j.jebdp.2005.12.016

[7] Boyer-Kassem T. Is the precautionary principle really incoherent? Risk Anal 2017; 37(11):2026-34. https://doi.org/10.1111/risa.12774

[8] Zhu N, Zhang D, Wang W, Li X, Yang B, Song J, et al. A novel coronavirus from patients with pneumonia in China, 2019. N Engl J Med 2020; 382(8):727-33. https://doi.org/10.1056/NEJMoa2001017

[9] de Souza Ferreira LP, Valente TM, Tiraboschi FA, da Silva GPF. Description of Covid-19 Cases in Brazil and Italy. SN Compr Clin Med 2020; 2020:1-4. https://doi.org/10.1007/s42399-020-00307-y

[10] Team CC-R. Characteristics of health care personnel with COVID-19 - United States, February 12-April 9, 2020. MMWR Morb Mortal Wkly Rep 2020; 69(15):477-81. https://doi.org/10.15585/mmwr.mm6915e6

[11] World Health Organization (WHO). Coronavirus disease (COVID-2019) situation reports 2020. Available from: https://www.who.int/emergencies/diseases/novel-coronavirus-2019/situation-reports. [Accessed on May 31, 2020].

[12] Rezende LFM, Thome B, Schveitzer MC, Souza-Júnior PRB, Szwarcwald CL. Adults at high-risk of severe coronavirus disease-2019 (Covid-19) in Brazil. Rev Saude Publica 2020; 54:50.

https://doi.org/10.11606/s1518-8787.2020054002596 
[13] Nussbaumer-Streit B, Mayr V, Dobrescu AI, Chapman A, Persad E, Klerings I, et al. Quarantine alone or in combination with other public health measures to control COVID-19: a rapid review. Cochrane Database Syst Rev 2020; 4:CD013574. https://doi.org/10.1002/14651858.CD013574

[14] USA. Department of Health \& Human Services. Community-Based Testing Sites for COVID-19. Available from: https://www.hhs.gov/coronavirus/community-based-testing-sites/index.html. [Accessed on May 30, 2020].

[15] Germany. Press and Information Office of the Federal Government. Measures taken by the German government to stem the COVID-19 pandemic. Available from: https://www.bundesregierung.de/breg-en/search/gegen-coronapandemie-1753880. [Access on June 11, 2020].

[16] Prather KA, Wang CC, Schooley RT. Reducing transmission of SARS-CoV-2. Science 2020 ; eabc6197. https://doi.org/10.1126/science.abc6197

[17] World Health Organization (WHO). When and how to use masks. Available from: https://www.who.int/emergencies/diseases/novel-coronavirus-2019/advice-for-public/when-and-how-to-usemasks. [Accessed on May 31, 2020].

[18] Centers for Disease Control and Prevention (CDC). How to Protect Yourself \& Others. Available from: https://www.cdc.gov/coronavirus/2019-ncov/prevent-getting-sick/prevention.html. [Accessed on May 31, 2020].

[19] Dixon DL, Van Tassell BW, Vecchié A, Bonaventura A, Talasaz AH, Kakavand H, et al. Cardiovascular considerations in treating patients with coronavirus disease 2019 (COVID-19). J Cardiovasc Pharmacol 2020; 75(5):359-67. https://doi.org/10.1097/FJC.0000000000000836

[20] Xu J, Li Y, Gan F, Du Y, Yao Y. Salivary glands: potential reservoirs for COVID-19 asymptomatic infection. J Dent Res 2020: 22034520918518. https://doi.org/10.1177/0022034520918518

[21] Centers for Disease Control and Prevention (CDC). Guidance for Dental Settings. Available from: https://www.cdc.gov/coronavirus/2019-ncov/hcp/dental-settings.html\#Management. [Accessed on May 31, 2020].

[22] Brasil. Secretaria de Atenção Primária à Saúde. Atendimento Odontológico no SUS. NOTA TÉCNICA No 9/2020$\mathrm{CGSB} / \mathrm{DESF} / \mathrm{SAPS} / \mathrm{MS}$, março de 2020. Brasília, 2020. Avaliable from: http://www.crosp.org.br/uploads/arquivo/ab69d79b87d04780afo8a70d8cee9d70.pdf. [Accessed on June 11, 2020]. [In Portuguese]

[23] Brasil. Agência Nacional de Vigilância Sanitária. NOTA TÉCNICA GVIMS/GGTES/ANVISA No 04/2020, de 08 de maio de 2020. Brasília, $2020 . \quad$ Avaliable from: http://portal.anvisa.gov.br/documents/33852/271858/Nota+T\%C3\%A9cnica+n+04-2020+GVIMS-GGTESANVISA/ab598660-3de4-4f14-8e6f-b9341c196b28. [Accessed on June 11, 2020]. [In Portuguese]

[24] Pereira LJ, Pereira CV, Murata RM, Pardi V, Pereira-Dourado SM. Biological and social aspects of Coronavirus Disease 2019 (COVID-19) related to oral health. Braz Oral Res 2020; 34:e041.

https://doi.org/10.1590/1807-3107bor-2020.vol34.0041

[25] American Dental Association (ADA). Return to Work Interim Guidance Toolkit (Updated 5/7/2020). Available from: $\quad$ https://success.ada.org/en/practice-management/patients/infectious-diseases-2019-novelcoronavirus?utm_source=adaorg\&utm_medium $=$ globalheader\&utm_content=coronavirus\&utm_campaign=covid19. [Accessed on June 1st, 2020].

[26] Clarkson J, Ramsay C, Aceves M, Brazzelli M, Colloc T, Dave M, et al. Recommendations for the re-opening of dental services: a rapid review of international sources. Available from: https://oralhealth.cochrane.org/news/recommendations-re-opening-dental-services-rapid-review-internationalsources. [Accessed on June 11, 2020]. 